\title{
Upgraded to Obsolescence: Age Intervention in the Era of Biohacking
}

\author{
KIRSTEN L. ELLISON ${ }^{1}$ \\ TRENT UNIVERSITY
}

\begin{abstract}
Popularized by DIY scientists and quantified-selfers, the language of "biohacking" has become increasingly prevalent in anti-aging discourse. Presented with speculative futures of superhuman health and longevity, consumers and patients are invited to "hack" the aging process, reducing age to one of the many programs, or rather "bugs" that can be re-written, removed, and rendered obsolete. Drawing on recent examples from popular media and anti-aging promotional materials, I explore how the language of biohacking signals an orientation to the body that denies the acceptability of a body that is anything but optimal. In the endless strive towards the latest and greatest, the language of biohacking renders the old body obsolete, standing as nothing more than a relic of an outdated operating system.
\end{abstract}

\section{Keywords}

biohacking; anti-aging; language; the body

In December of 2015, I attended the American Association of Anti-Aging Medicine's 23rd annual meeting in Las Vegas. While walking through the Exhibit Hall lined with vendor booths for hydrotherapy, cryotherapy, stem cell therapy, oxygen therapy, electromagnetic therapy, heat therapy, hormone therapy, thermo-imaging, neuro-imaging, and various skin creams, serums, and treatments, I came across a booth for Bulletproof coffee. Perplexed as to the role of coffee in age intervention, I asked the vendor what his product was all about. Coffee, he explained, is the gateway drug to biohacking your body. As a communications scholar with an interest in representations of the body, the metaphor of the body as a computer system was not anything new. In popular coverage of developments in age intervention, genes are often described as being

\footnotetext{
${ }^{1}$ Kirsten Ellison, Email: kellison@trentu.ca

Copyright (C) 2020 (Kirsten Ellison). Licensed under the Creative Commons Attribution Non-commercial No Derivatives (by-nc-nd). Available at estsjournal.org.
} 
switched on or off, enzymes activated and boosted, bodies rebooted, biochemistries reprogrammed, and overall bodily function upgraded and optimized. These verbs are frequently employed to describe techniques of anti-aging at the molecular level. But the language of hacking - and specifically biohacking - is a relatively new and different extension of the computer metaphor to age intervention. As an overarching language of intervention, the recent surge in popularity and commercialization of biohacking aligns a diverse range of practices and techniques along a common goal orientation of optimization, with the old body standing as nothing more than a relic of an outdated operating system. As I argue below, this logic of intervention has significant implications for how we understand our own aging bodies and the malleability of our vital futures.

Merriam-Webster defines hacking as the gaining of illegal access to a computer system or network. Hackers, in this sense, are working against the status quo, rogue operators who actively seek to circumvent the rules and take control of the system for their own gains. Biohacking, in this context, refers to the gaining of unconventional access to the body and its biological mechanisms, circumventing the "rules" of biology, and breaking into what is understood here as the "software of life." In his book, Biopunk: DIY Scientists Hack the Software of Life, Marcus Wohlsen describes the work of do-it-yourself scientists and biologists working against the "system" in back rooms, basements and garages with the goal of empowering individuals by open-sourcing the genetic code, understood here as the code of life. Biohacking refers in this case to the democratization of the body and its functions at the level of the genome. In the context of age intervention, however, and the broader goals of personal health and optimization, biohacking refers instead to the personal tailoring of intervention and consumption, where the hacker actively engages in the commodified pursuit of optimal performance.

This version of "biohacking," as both noun and verb, was popularized and commercialized by Dave Asprey. His company, Bulletproof ${ }^{\circledR}$, is a nutritional supplement and selfhelp enterprise, which includes a line of Bulletproof ${ }^{\mathrm{TM}}$ and Upgraded $^{\mathrm{TM}}$ products and services such as coffee shops, life and cooking manuals, webinars, conferences, laboratories, food and beverage products, nutritional supplements, and more. According to Asprey, biohacking refers to both "the art and science of becoming superhuman" and the use of "systems thinking, science, biology, and self-experimentation to take control and upgrade your body, your mind, and your life." To this, Asprey adds a definition of "bulletproof" as the "state of high performance, resilience and vibrant health where your body, mind, and life work together in unison, providing performance beyond what you'd expect." In the webpage's accompanying infographic, aging is but one of many nodal points in the biology of the body that can be "hacked." Online, many of 
the company's blogs, videos and podcasts are devoted to the topic of anti-aging. In one blog, they list the "Top anti-aging biohacks for better skin." Included in this list of hacks is a peptide cream and injection, cryotherapy, microneedling, and stem cell therapy.

The literal sense of the biohacker as both bulletproof and superhuman finds its most extreme expression in Finland's Biohacker Center's promotional materials for its annual summit. In 2014, the Center released a promotional video with the torso of a young man in a suit, opening his shirt to reveal not the crest of Superman, but an entryway into the molecular, genetic and quantified structures of his body. Moving through the "software" of the inner body, the video reads: "It is time to take it to the next level. With quantified self and biohacking, you can be a better version of yourself." Calling to the power of a biotech singularity, where technology and biology "will unite," the video ends with the imperative to "upgrade yourself." And in the 2018 promotional video for the Biohacker Summit that was held Toronto, "biohacking the aging code" is listed as one of the key outcomes of the "biohacking revolution," alongside activating human performance, maximizing genetic potential, and increasing quality of life. Echoing this, Swiss think tank, Gottlieb Duttweiler Institute of Economic and Social Studies, in partnership with the Global Wellness Institute, released a report in 2018 identifying "biohacking" as a key trend in the future of the wellness industry. Biohackers, they explain, are at the forefront of wellness, "a subculture of people with a whole range of different backgrounds," "who want to liberate themselves from the limitations of nature, age and disease." "From the perspective of a biohacker," the report states, "immortality is an engineering problem" (p. 18). And as one American-based anti-aging clinic claims, "We want to bring you not only to health, but to optimal. We will help you to "hack" your inner computer to get your systems functioning optimally. We call this bio-hacking."

These ideals align well with anti-aging medicine's pursuit of enhancement and perfection, where the goal of intervention is not the achievement of normal health and "healthy" aging, but the eradication of aging all together. As anthropologist Courtney Mykyntyn has observed in her paper, "Medicalizing the Optimal: Anti-aging medicine and the quandary of intervention," the drive to overcome aging is understood in this context as more natural than the process of aging itself. Optimal health in this sense is decoupled from chronological age, defined instead by the best you can be at any age. In striving towards optimal performance or functionality, what is deemed "natural" is often marked as underperformative and dysfunctional and in need of corrective measures of intervention and optimization (for a seminal work on the turn to functionality in the discourse of successful and healthy aging, see Katz and Marshall's article, "Is the Functional 'Normal'? Aging, Sexuality and the Bio-marking of Successful Living"). 
In the case of biohacking, the optimization of performance is not about fixing or replacing parts, but about upgrading the functioning of the system itself in order to move beyond normal, and beyond health.

The language of biohacking, however, does not signal new ways of intervening in the aging body; rather, it aligns a broad range of interventions along a common goal orientation of optimization. In February of 2017, Shape published an article titled "How to Hack your Telomeres to Slow Aging and Live Longer." Listed hacks include having sex often, eating Omega-3s, sleeping better, doing more cardio, de-stressing yourself, moving more, and clearing the air with plants and air purifiers and such. In July of 2017, Capital City Nurses posted a blog titled: "Hack the Aging Process," but here "hacking" is interpreted as creating life shortcuts through the use of technology to "simplify the aging process" - including the use of a wireless sensor system for the home (BeClose), a medication distribution machine (TabSafe), and an easy to manage touch screen tablet (Telikin). In February of 2018, Healthline posted an article titled "Why scientists think 'Hacking our Cells' could turn off the aging process." Hacking is interpreted in this case as the maintenance and improvement of the health and functioning of the mitochondria through exercise, diet, and supplements. In 2017, Popular Science has included an entire section of their special issue on The Science of Living Longer to "Hacking Longevity," and MIT Technology Review to "Hacking the Biological Clock." Even AARP published an article in 2018 titled "Hacking longevity: Understanding the implications of living to 100." Hacking in these cases is used in reference to cybernetics, cryogenics, blood transfusions, precision medicine, gene editing, calorie restriction, and the drugs mimicking effects of calorie restriction (like rapamycin), brain uploading, egg rejuvenation, and the list goes on. And in British Columbia, Canada, the health and wellness enterprise, Ageless Living, has devoted an entire treatment center to biohacking with treatments that include IV therapy (which is a customized vitamin therapy), NuCalm therapy (which is a machine that puts you into a state of transcendental meditation), PEMF (pulsed electromagnetic therapy), Cryotherapy, Hyperbaric Oxygen therapy, Neurointegrator (which the use of neurofeedback to retrain your brain), Infrared Sauna therapy, Hormone restoration, Hair loss therapy, Microneedling therapy, and a Juice Bar! (to help with fatigue and workout recovery).

It seems, from these examples, that anything can be interpreted as a hack to the aging process, so long as the drive remains the same: a will to move beyond health, taking the aging body to not only a younger state where it no longer exists, but to an optimal one where it perhaps never existed; a will to be better than normal, or as Asprey has put it, to be superhuman. In one of his seminal works, "The politics of life itself," Nikolas Rose argues that forward vision is one of 
the key features of what he terms technologies of optimization. In this case, the ethical subject, or rather, the bulletproof self, is defined not in terms of the end result of intervention, or some underlying and uncovered essence, but in terms of the work that is done in order to continuously maximize the constantly shifting standards of high performance, resilience and optimal health touted by the pioneers of the "biohacking revolution." Here, age is reduced to one of the many programs, or rather "bugs" that can be re-written, removed, and rendered obsolete. The "hacked body" in this sense is the perpetually youthful, functional, and ageless body. Just as with the "fit body," it is a state that can never be fully achieved and yet must constantly be striven for, a continuous propelling forward. As Asprey states on the Bulletproof ${ }^{\circledR}$ website, "No matter who you are, you can get even better."

In the past decade, increasing attention has been paid to the entanglements of aging, technology and science in the field of science and technology studies. In their chapter in the most recent Handbook of Science and Technology Studies, Kelly Joyce, Alexander Peine, Louis Nevin and Florian Kohlbacher argue for the need to take into account how problematic and largely ageist assumptions about older people and their bodies are interwoven into the design and rationale of technological innovations aimed at solving the "problems" of an aging population. What the present issue adds to this discussion is the importance of examining how the logic of technological fixes has entered into a common vernacular of intervention that includes everything from sleep and diet to heat therapy and neurotropic drugs. Rather than the techniques themselves, it is the transference of a logic of corporeal manifest destiny that constitutes the "biohacking universe" of contemporary anti-aging practices. Accordingly, the body is reduced to an ever-optimizable territory of colonization and its intervention justified by the pursuit and inevitability of technological progress. In the open horizons of the "hacked" body, aging is but a re-writable glitch in the ever-perfectible terrain of our optimal futures. Significant here is that aging is not merely understood as a "problem" to be fixed by technology, but its value and belonging in our shared corporeal destinies is trivialized, reduced to a biological fate that is no more inevitable than our B.M.I. Beyond the techniques themselves, we need to think critically about the language that drives intervention and the kinds of bodies that are rendered possible and desirable in the landscape of optimization. 


\section{Author Biography}

Kirsten Ellison is a Postdoctoral Fellow at Trent University, funded by the SSHRC Insight Grant, Digital Culture \& Quantified Aging. Her research examines the intersections of aging, science and technology in popular culture, with a focus on visual and linguistic representations of the body.

\section{Acknowledgements}

I would like to thank Drs. Barb Marshall and Stephen Katz for their feedback and encouragement in the development of the ideas presented here and the ESTS editors for their thoughtful comments and suggestions. 